University of New Hampshire

University of New Hampshire Scholars' Repository

Space Science Center

Institute for the Study of Earth, Oceans, and

Space (EOS)

5-30-2006

\title{
FNIT: the fast neutron imaging telescope for SNM detection
}

\author{
U Bravar \\ University of New Hampshire - Main Campus \\ P J. Bruillard \\ University of New Hampshire - Main Campus \\ E O. Fluckiger \\ University of Bern \\ John R. Macri \\ University of New Hampshire - Main Campus, John.Macri@unh.edu \\ Mark L. McConnell \\ University of New Hampshire - Main Campus, mark.mcconnell@unh.edu
}

See next page for additional authors

Follow this and additional works at: https://scholars.unh.edu/ssc

Part of the Astrophysics and Astronomy Commons

\section{Recommended Citation}

Ulisse Bravar ; Paul J. Bruillard ; Erwin O. Flückiger ; John R. Macri ; Mark L. McConnell ; Michael R. Moser and James M. Ryan "FNIT: the fast neutron imaging telescope for SNM detection", Proc. SPIE 6213, NonIntrusive Inspection Technologies, 62130G (May 30, 2006); doi:10.1117/12.666119; http://dx.doi.org/ $10.1117 / 12.666119$

This Conference Proceeding is brought to you for free and open access by the Institute for the Study of Earth, Oceans, and Space (EOS) at University of New Hampshire Scholars' Repository. It has been accepted for inclusion in Space Science Center by an authorized administrator of University of New Hampshire Scholars' Repository. For more information, please contact Scholarly.Communication@unh.edu. 


\section{Authors}

U Bravar, P J. Bruillard, E O. Fluckiger, John R. Macri, Mark L. McConnell, M R. Moser, and James M. Ryan 


\title{
FNIT: the Fast Neutron Imaging Telescope for SNM Detection
}

\author{
Ulisse Bravar ${ }^{*}$, Paul J. Bruillard ${ }^{\mathrm{a}}$, Erwin O. Flückiger ${ }^{\mathrm{b}}$, John R. Macri ${ }^{\mathrm{a}}$, Mark L. McConnell ${ }^{\mathrm{a}}$, \\ Michael R. Moser ${ }^{\mathrm{b}}$, James M. Ryan ${ }^{\mathrm{a}}$ \\ ${ }^{a}$ Space Science Center, University of New Hampshire, 39 College Road, Durham, NH 03824, USA \\ ${ }^{\mathrm{b}}$ Physikalisches Institut, University of Bern, Sidlerstrasse 5, CH-3012 Bern, Switzerland
}

\begin{abstract}
We report on recent progress in the development of the Fast Neutron Imaging Telescope (FNIT), a detector with both imaging and energy measurement capabilities, sensitive to neutrons in the 2-20 MeV range. FNIT was initially conceived to study solar neutrons as a candidate design for the Solar Sentinels program under formulation at NASA. This instrument is now being configured to locate fission neutron sources for homeland security purposes. By accurately identifying the position of the neutron source with imaging techniques and reconstructing the energy spectrum of fission neutrons, FNIT can locate problematic amounts of Special Nuclear Material (SNM), including heavily shielded and masked samples. The detection principle is based on multiple elastic neutron-proton (n-p) scatterings in organic scintillators. By reconstructing the $n-p$ event locations and sequence and measuring the recoil proton energies, the direction and energy spectrum of the primary neutron flux can be determined and neutron point sources identified. The performance of FNIT is being evaluated through a series of Monte Carlo simulations and lab tests of detector prototypes. The Science Model One (SM1) of this instrument was recently assembled and is presently undergoing performance testing.
\end{abstract}

Keywords: neutron imaging, neutron spectroscopy, scintillation detectors, SNM detection, wavelength shifting fibers

\section{INTRODUCTION}

A critical gap in national security is the inability to efficiently detect and identify problematic quantities of Special Nuclear Material (SNM). These materials, specifically uranium and transuranics, emit neutrons via spontaneous or induced fission. Unlike the other forms of radiation produced by SNM (e.g. $\gamma$-rays), copious and penetrating neutron emission is unique to fissionable material. From a practical point of view, shielding of fission neutrons (e.g. in a cargo container) represents a far greater challenge and requires a considerably larger and heavier amount of passive material than shielding of other forms of radiation emitted by SNM. Neutron detection, therefore, is of particular interest for SNM identification for security and proliferation deterrence, as well as for nuclear waste detection and monitoring.

While improvements in all forms of radiation detection are necessary to close the SNM security gap, there are unique problems associated with the detection and measurement of neutrons. Some of these are:

- current neutron detectors used in the field (e.g. Bonner spheres ${ }^{1}$ ) have not changed significantly in decades;

- current neutron detectors do not directly detect the fission neutrons, but rather register their presence only after moderation, after they have lost all original energy and directional information;

- current neutron detectors do not image the neutron source; and

- current neutron detectors provide energy information only for the case of high intensities or long exposures.

The Fast Neutron Imaging Telescope (FNIT) was first conceived by an international team as a candidate instrument for the Solar Sentinels ${ }^{2}$ program, presently in the formulation stage at NASA, to be deployed on a spacecraft to the inner heliosphere and study neutrons from solar flares in the 2-20 MeV range. However, it was soon realized that the design characteristics of FNIT, namely its spectroscopy and imaging capabilities in this energy range, make it a powerful tool in the search for SNM samples. The FNIT instrument is designed to locate a neutron point source by imaging alone. In addition, a fission spectrum measured by FNIT would represent a clear signature of SNM. While the basic design of

*Ulisse.Bravar@unh.edu; phone 1 (603) 862-3868; fax 1 (603) 862-3584

Non-Intrusive Inspection Technologies, edited by George Vourvopoulos, F. Patrick Doty, Proc. of SPIE Vol. 6213 62130G, (2006) - 0277-786X/06/\$15 - doi: 10.1117/12.666119

Proc. of SPIE Vol. 6213 62130G-1 
FNIT makes no distinction between space and ground-based applications, there are unique requirements that need to be met in order to configure this detector as a field-deployable instrument for SNM identification.

\section{SNM NEUTRONS}

Currently, neutron counters used in SNM surveys do not directly detect the fission neutrons, but rather register their presence only after moderation, i.e. after they have lost all original energy and directional information. Because of this limitation and the presence of the atmospheric neutron background, current detection systems often do not have sufficient sensitivity to detect problematic and dangerous amounts of fissionable material. The count rate from fission neutrons at meter distances from a SNM sample is comparable to the atmospheric neutron background flux and all the information necessary to identify a SNM signature by reconstructing its fission energy spectrum is missing.

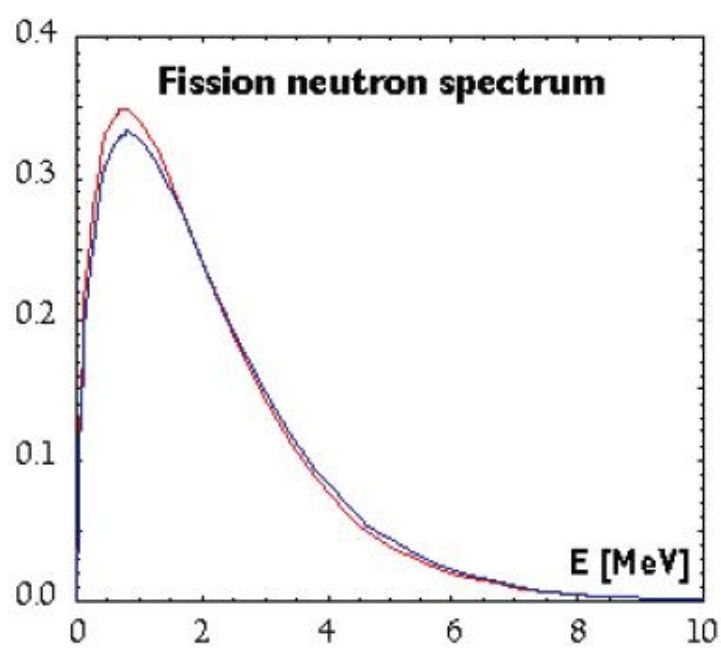

Figure 1. Fission neutron energy spectra for ${ }^{239} \mathrm{Pu}$ (blue) and ${ }^{235} \mathrm{U}$ (red).
To put these observations into perspective, consider a typical portable neutron detector, $10 \times 10 \mathrm{~cm}^{2}$ in active area and with a $10 \%$ efficiency, placed at a $10 \mathrm{~m}$ distance from a $1 \mathrm{~kg}$ sample of Weapon Grade Plutonium (WGP). One kilogram of WGP emits on average $6 \times 10^{4}$ neutrons/s and their energy spectrum follows the Watt distribution ${ }^{3}$ shown in Figure 1, with a peak at $1 \mathrm{MeV}$ and a mean value of $2 \mathrm{MeV}$. The sea-level atmospheric neutron flux at energies below $10 \mathrm{MeV}$, calculated from recently published data ${ }^{4}$, is on average $\sim 5 \times 10^{-3}$ neutrons $/ \mathrm{cm}^{2} \mathrm{~s}$, with large time excursions and a rapid increase with altitude. Simple algebra shows that the detector in this example would record 0.05 neutrons/s from atmospheric background and an additional 0.05 neutrons/s from the WGP. Imaging would therefore play a crucial role in successfully identifying and locating this SNM sample.

The neutron yield from uranium is several orders of magnitude smaller than the emission from WGP and the only practical method of identifying kilogram quantities of Highly Enriched Uranium (HEU) is through active interrogation. Past tests using active interrogation techniques demonstrated the feasibility of detecting gram-size amounts of this material by placing neutron counters at distances of $\sim 0.1 \mathrm{~m}$ from unshielded HEU samples ${ }^{5}$. However, a rapid degradation in performance was noticed when increasing the SNM sample detector distance. The extension to $\mathrm{kg}$-size samples and $\sim 10 \mathrm{~m}$ distances, combined with the addition of shielding material, requires the introduction of neutron imaging. In summary, due to the limited performance of present-day state of the art neutron detectors, the inability to search for and find clandestine amounts of SNM is a major shortcoming in nuclear security systems. Detector sensitivities need to be improved at a minimum by one to two orders of magnitude to introduce a significant change to this situation.

\section{FISSION NEUTRON DETECTION}

Because they are electrically neutral, neutrons must be detected using indirect means. The preferred method at $\mathrm{MeV}$ energies takes advantage of the fact that the neutron-proton ( $n-p)$ elastic scattering cross section is large. The recoil

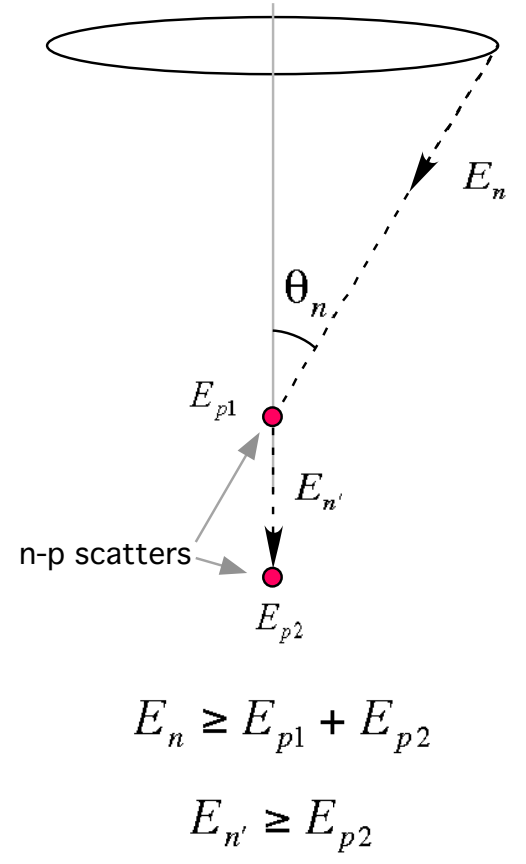

Figure 2. Double elastic n-p scattering. Schematic showing the basic kinematics of event reconstruction. If the full neutron energy is measured, the incident neutron arrival direction is restricted to the mantle of a cone and an "event circle" can be drawn. 
proton from an n-p scatter is a highly ionizing particle and is easily detected with a suitable instrument. To employ this technique in its full potential, it is advantageous to have neutrons scatter off protons rather than heavier nuclei. Therefore, an ideal material, acting both as neutron scatterer and recoil proton detector, is organic scintillator (plastic of liquid $)^{1}$. In a plastic scintillator plate, light produced by the ionization energy loss of recoil protons can be measured with appropriate optoelectronics (e.g. photomultiplier tubes) and its intensity related to the kinetic energy of the scattered proton. This type of detector has a number of desirable features. It is compact, lightweight, fast and low-cost. In its basic form, however, it is omni-directional, and therefore cannot achieve the levels of sensitivity required for neutron source characterization. A directional, or better still, an imaging scintillator detector can be far more selective and discriminating against background, yielding much improved sensitivity.

To perform imaging and reconstruct the energy of the incident neutron, it is necessary for this particle to undergo several (at least two) elastic n-p scatterings in the detector. One must be able to follow the path of the scattered neutron and to clearly identify and separate individual $n-p$ interactions, measuring the coordinates, relative time and recoil proton energy after each n-p collision.

Consider the case shown in Figure 2. A neutron, whose incident direction and energy are unknown, undergoes two n-p scatterings. From the coordinates and relative times of the two interactions, one can determine the time of flight (ToF) and direction of the scattered neutron. By computing the energy of the scattered neutron from its ToF and measuring the energy of the first recoil proton from scintillation light yield, one can then determine the energy of the incident particle. With this information, the neutron scatter angle $\theta_{\mathrm{n}}$ can be derived from the simple kinematics of elastic nonrelativistic scattering as:

$$
\sin ^{2} \theta_{n}=\frac{E_{p 1}}{E_{n}}
$$

where $E_{\mathrm{p} 1}$ and $E_{n}$ are the kinetic energies of the first recoil proton and the incident neutron, respectively ${ }^{1}$. Additionally, if a third n-p interaction is observed, the coordinates of the third scattering provide the additional kinematic information necessary to uniquely define the incident neutron energy and scatter angle $\theta_{\mathrm{n}}$, making the ToF measurement redundant.

Data from two or more $n-p$ interactions is sufficient to constrain the direction of the incident neutron onto the mantle of a cone about the recoil neutron velocity vector. At this stage a point source still needs to be identified. In general, if the velocity vector of the first recoil proton were known, having determined the energy and track of the recoil neutron in the previous paragraph, the exact direction of the incident neutron would be fixed by linear momentum conservation. However, at FNIT energies the recoil proton cannot be tracked due to its short range in scintillator and one can only measure the proton's kinetic energy. However, by projecting the incident neutron cone onto a plane in the far-field, one can define an "event circle" for this particle, visible at the top of Figure 2.

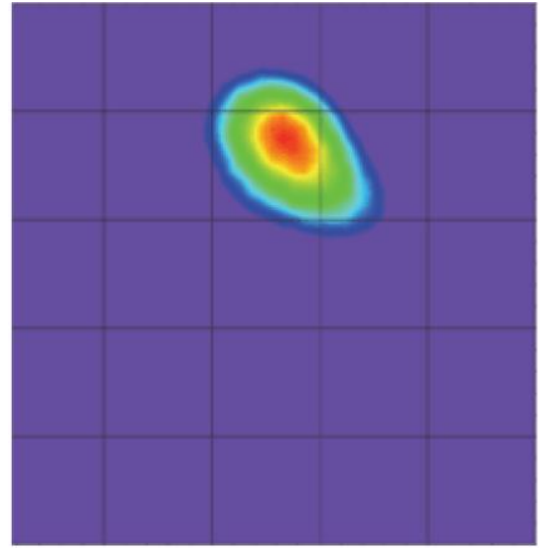

Figure 3. Demonstration of the "event circle" imaging technique: neutron image of the $\mathrm{Sun}^{7}$.

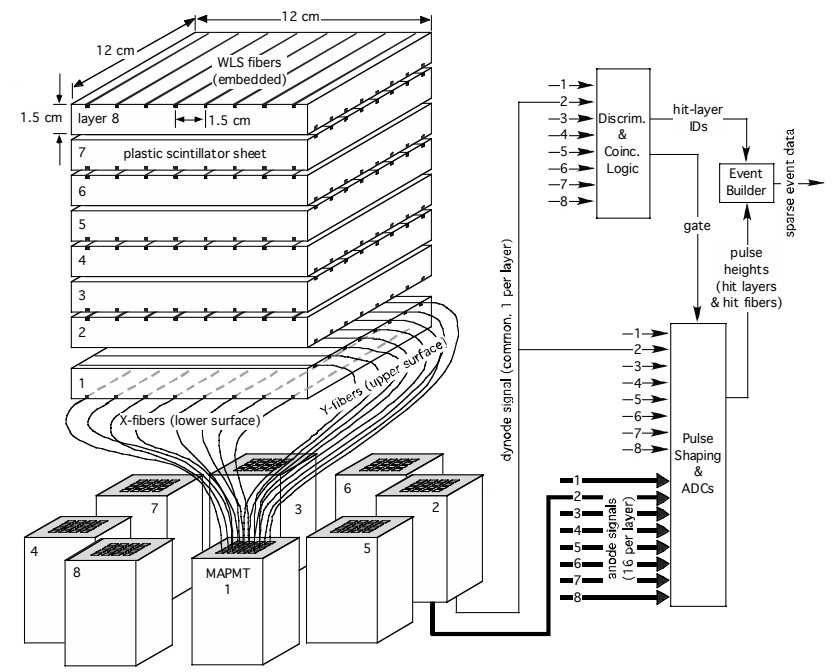

Figure 4. Conceptual design of FNIT, consisting of a tower of eight position sensitive scintillator detector modules, WLS fibers coupled to MAPMTs and readout electronics. For clarity, only 16 WLS fibers per module are represented in this figure, the actual number being 64. Presently, a spacing of $3.0 \mathrm{~cm}$ between layers is used. 
By collecting a sufficiently large population of neutron events, it becomes possible to determine the point where individual event circles intersect and use it to mark the position of the neutron source. This same principle is presently employed for $\gamma$-ray imaging in Compton telescopes. Namely, event circles from a source intersect at a specific point, while the imaging of an isotropic background leads to circles with isotropically distributed intersections. The effectiveness of the event-circle technique was also demonstrated in the case of neutrons by reconstructing the image of the Sun shown in Figure 3. This image was generated from a sample of $\sim 80$ solar neutrons in the $20-80 \mathrm{MeV}$ energy range, measured by the COMPTEL instrument ${ }^{6}$ during the 15 June 1991 solar flare ${ }^{7}$

\section{THE FAST NEUTRON IMAGING TELESCOPE}

The baseline design of FNIT, consisting of a tower of eight detector modules, is shown in Figure 4. Central to each module is a sheet of plastic scintillator. Grooves are machined into both surfaces of the sheet with a regular pitch and orthogonal orientations on the two sides. A wavelength shifting (WLS) plastic fiber is bonded into each groove and routed to one pixel on the face of a multianode photomultiplier tube (MAPMT). A small portion of the blue scintillation light created in the plastic scintillator is absorbed by the WLS fibers on both surfaces close to the recoil proton position and re-emitted as green light. The clad WLS fibers act as light guides for the trapped portion of this green light signal that travels the length of the fiber to the MAPMT pixel for readout. Each detector module is coupled to its own 16-channel MAPMT and the instrument trigger is a coincidence of signals from two or more modules. When triggered, the IDs and pulse heights of the light signals from each WLS fiber are time-tagged and read out as event messages for storage and further processing. The energy and coordinates of each recoil proton are reconstructed from pulse height analysis. Finally, the measurement of the relative timing of signals is used to determine the interaction sequence and ToF of scattered neutrons.

The detailed design of the individual detector module was the subject of a lengthy optimization process described elsewhere ${ }^{8,9}$. We experimented with different detector dimensions, combined with a selection of scintillator coatings and WLS fiber pitches, using both round and square WLS fibers. We performed a series of Monte Carlo simulations to select the best thickness and efficiency for the individual scintillator sheet, fabricated several prototype modules with different design characteristics and conducted a number of benchmark measurements (light output, spatial, time and energy resolution) to finalize the detector module design.

At the conclusion of this process, the following design parameters of the detector module were frozen and selected for use in further implementations ${ }^{8,9}$ :

- BC-404 plastic scintillator sheet ${ }^{10}, 12.0 \mathrm{~cm} \times$ $12.0 \mathrm{~cm} \times 1.5 \mathrm{~cm}$ (thick);

- 64 evenly-spaced, equal length BCF-91A multiclad WLS round fibers ${ }^{10},(32 \mathrm{x}$-fibers and $32 \mathrm{y}$ fibers), diameter $=0.1 \mathrm{~cm}$, pitch $=0.375 \mathrm{~cm}$;

- one Hamamatsu H8711-10, 16 channel MAPMT $^{11}(4 \times 4$ array);

- bundles of four fibers each routed to individual MAPMT pixels (i.e. 64 fibers feeding 16 MAPMT channels);

- localized reflective coating (aluminized mylar) of the two $12 \times 12 \mathrm{~cm}^{2}$ surfaces of the scintillator sheet;

- black coating to cover the edge surfaces of the scintillator sheet;

- polished aluminum end-mirrors to terminate the unused ends of the WLS fibers and reflect the

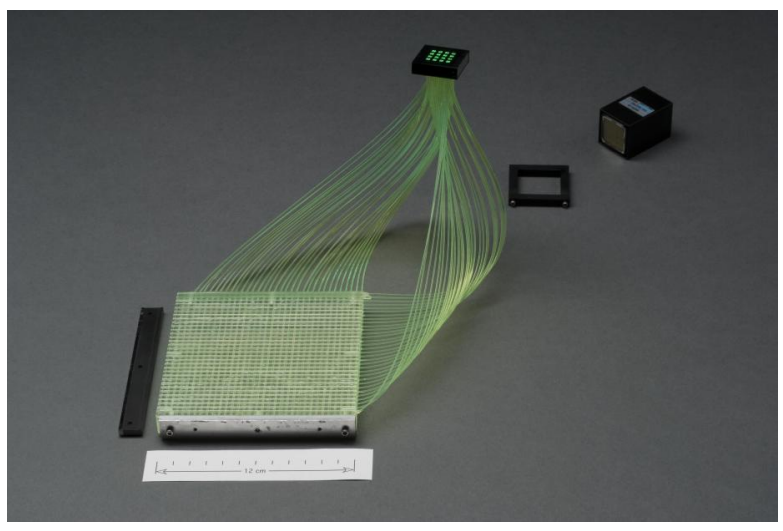

Figure 5. FNIT prototype detector module. The plastic scintillator, $12 \times 12 \mathrm{~cm}^{2}$ in area, is unwrapped. Two black terminators are visible next to the right and bottom scintillator edges. The 64 WLS fibers form an $x-y$ mesh in the scintillator and are grouped into a $4 \times 4$ array of 16 pixels at one of the extremities. The 16-pixel array is coupled to the MAPMT shown in the top right corner of this image. 
light signal back to the MAPMT; and

- minimum practically achievable WLS fiber length $(\approx 20 \mathrm{~cm})$ between scintillator and photocathode.

A photograph of this detector module is shown in Figure 5. The effective pitch of 4-fiber bundles coupled to each MAPMT channel $(0.375 \mathrm{~cm} \times 4=1.5 \mathrm{~cm})$ is equal to the thickness of the scintillator sheet. A list of the possible options for each design parameter and the rationale behind each chosen value are described in detail elsewhere ${ }^{8,9}$.

The performance of the selected detector module design was characterized in a full set of lab tests using cosmic-ray muons, plus $\beta$ - and $\gamma$-ray lab sources. We measured the following values for the principle performance parameters:

- energy resolution $\delta \mathrm{E} / \mathrm{E}=16 \%$ at $2.6 \mathrm{MeV}_{\text {ee }}$;

- time resolution $\delta \mathrm{t}=0.6 \mathrm{~ns}$ at $2.6 \mathrm{MeV}_{\mathrm{ee}}$;

- horizontal position resolution $\delta \mathrm{x} \approx \delta \mathrm{y} \approx 0.85 \mathrm{~cm}$ at $2 \mathrm{MeV}_{\mathrm{ee}}$;

- $\quad$ light yield = 40 photoelectrons $/ \mathrm{MeV}_{\mathrm{ee}}$; and

- $\quad$ energy threshold $=0.125 \mathrm{MeV}_{\mathrm{ee}}$.

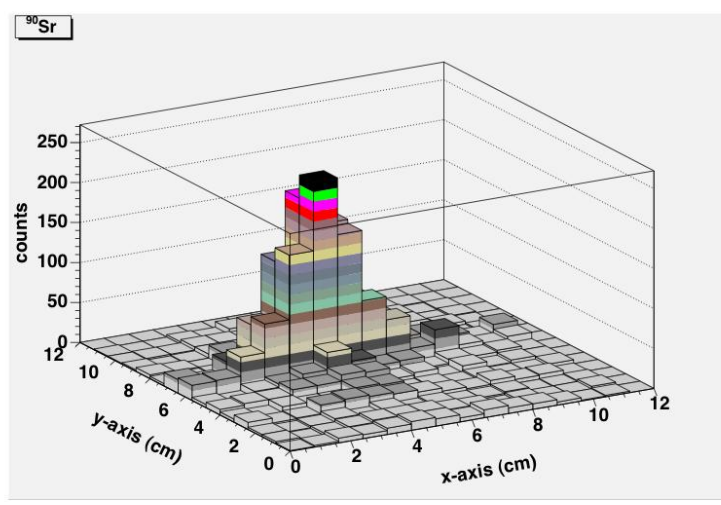

Figure 6. Horizontal position resolution of the FNIT prototype detector module. Reconstructed position in the $\mathrm{x}$ and $\mathrm{y}$ horizontal coordinates of a needle $\beta$-beam from a collimated ${ }^{90} \mathrm{Sr}$ source irradiating one side of the scintillator sheet. The differences in $\delta x$ and $\delta y$ are clearly visible.

All the tabulated resolutions are r.m.s. values and all the energies are electron equivalent figures (hence the subscript ee borne by all $\mathrm{MeV}$ labels). At $\mathrm{MeV}$ energies, an electron and a proton with the same kinetic energy result in significantly different numbers of scintillation photons. In particular, in the BC-404 scintillator, a $1 \mathrm{MeV}$ electron translates into a six times larger light yield than a $1 \mathrm{MeV}$ proton $^{10}$. We used electrons and particles producing electron-like signals in our lab tests due to limited equipment availability. Neutrons however are detected through the signal from recoil protons. In particular, the quoted energy threshold of $0.125 \mathrm{MeV}_{\text {ee }}$ translates into a low-limit value of $6 \times 0.125 \mathrm{MeV}=$ $0.75 \mathrm{MeV}$ for protons. At a minimum, two recoil protons are needed to detect a neutron. Therefore, the neutron threshold achievable by our detector module design can be conservatively assumed to lie at around $2 \mathrm{MeV}$. Lowering the threshold value may be the crucial factor in further adapting FNIT to SNM detection.

Figure 6 illustrates the horizontal position resolution of the detector module determined from lab data. A collimated ${ }^{90} \mathrm{Sr} \beta$-source was placed on one of the sides of the scintillator sheet and the two coordinates of each electron of this needle beam were reconstructed from the signals recorded by the MAPMT. Earlier, we reported an average position resolution of $0.85 \mathrm{~cm}$. In reality, the two resolutions measured from Figure 6 are $\delta x=1.25 \mathrm{~cm}$ and $\delta y=0.45 \mathrm{~cm}$. Why are these two values so different? The answer lies in the fact that the range of the ${ }^{90} \mathrm{Sr} \beta$-electrons is shorter than the thickness of the scintillator sheet and electrons undergo multiple scattering before stopping. Yfibers were grooved onto the irradiated side of the scintillator, while $\mathrm{x}$-fibers were coupled to the opposite side. As a consequence, the light collected by the $y$-fibers was more localized than the light seen by the $\mathrm{x}$-fibers.

\section{FNIT PERFORMANCE SIMULATION}

A Monte Carlo simulation code based on the GEANT4 ${ }^{12}$

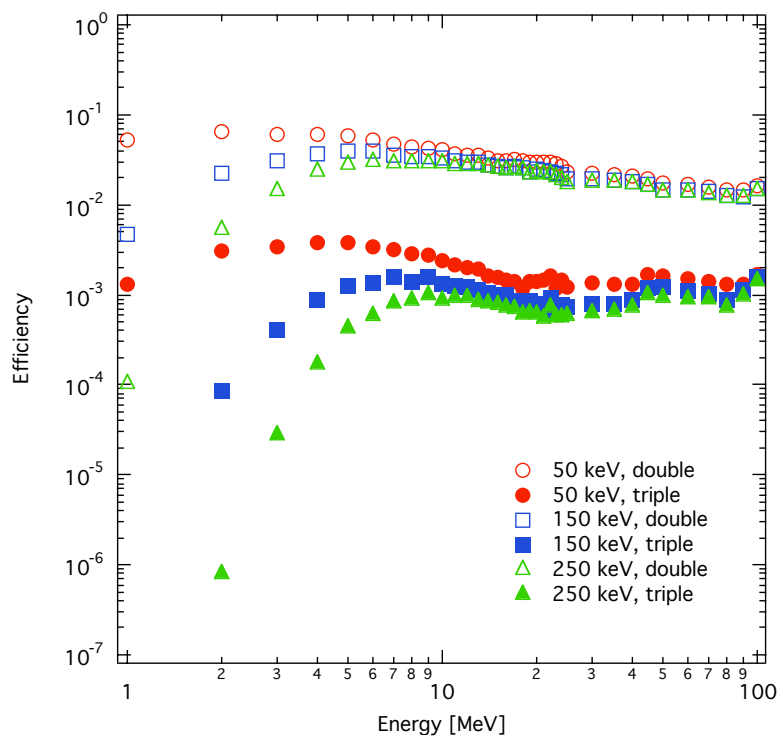

Figure 7. Efficiency of the FNIT instrument as a function of the incident neutron energy, determined by Monte Carlo simulations. Efficiencies for double and triple n-p scatter events are represented separately and results for three values of the energy threshold $\left(0.05 \mathrm{MeV}_{\mathrm{ee}}, 0.15 \mathrm{MeV}_{\mathrm{ee}} \& 0.25 \mathrm{MeV}_{\mathrm{ee}}\right)$ are shown. 
package was developed to support the FNIT design effort. It was used to optimize the configuration of FNIT and determine its overall efficiency. A full simulation of actual application scenarios with neutrons from SNM samples blended with atmospheric background neutrons and other ionizing particles, as well as the development and testing of the analysis software to image neutron point sources are pending tasks at the time of writing. The parameters on detector module resolution presented in the previous section, together with the differences in signal intensity when comparing electrons to protons, were incorporated into the Monte Carlo code prior to running the simulations presented underneath.

The sensitivity of the FNIT tower structure described in Section 4 , consisting of 8 detector modules, $12 \mathrm{~cm} \times 12 \mathrm{~cm} \times 1.5 \mathrm{~cm}$ in size, determined with this Monte Carlo code, is presented in Figure 7. Efficiencies of up to $50 \%$ are achieved for single n-p scatter events and a peak efficiency of $10 \%$ was observed for double n-p scatters. This peak efficiency is strongly energythreshold dependent, especially at lower energies. The measured threshold value presented in the previous section, 125 $\mathrm{MeV}_{\text {ee }}$, translates into an efficiency of $2-3 \%$ at $2 \mathrm{MeV}$ incident neutron energy for double n-p scatter events. Figure 7 also shows that neutrons can be detected with slightly decreased performance to energies of over $100 \mathrm{MeV}$. While FNIT was conceived from the beginning to operate at neutron energies $\mathrm{E}<$ $20 \mathrm{MeV}$, this extended range provides critical cross-calibration capability with existing neutron imaging detectors for space that were developed for higher energies ${ }^{13}$.

Next, Monte Carlo data were used to determine the energy resolution for neutrons. The FNIT instrument was irradiated with $0-30 \mathrm{MeV}$ neutrons and the energy of each double and triple n-p scatter event was calculated using the principles described in Section 3. Four different energy reconstruction algorithms were evaluated, each of them based on a different combination of $n-p$ scattering coordinates, recoil proton energies and scattered neutron ToF information. Then, the reconstructed energy was compared to the actual energy of the neutron on an event-by-event basis. Figure 8 presents the results of this study. The r.m.s. energy resolution of FNIT at neutron energies below $5 \mathrm{MeV}$ was determined to be $\delta \mathrm{E} / \mathrm{E}=35 \%$.

This set of neutron data was also used to estimate angular resolution. The neutron scatter angle $\theta_{\mathrm{n}}$ for double and triple np scatters, defined in Figure 2, was reconstructed on an eventby-event basis and the reconstructed value of $\theta_{\mathrm{n}}$ was compared to its actual value. Results are illustrated in Figure 9. An r.m.s. angular resolution of $\delta \theta_{n}=10^{\circ}$ was achieved. Given such a resolution, how well can we locate a neutron point source and how many neutron events (i.e. how long of an exposure) are needed to clearly identify such a source? This and similar questions will be addressed in our future modeling efforts.

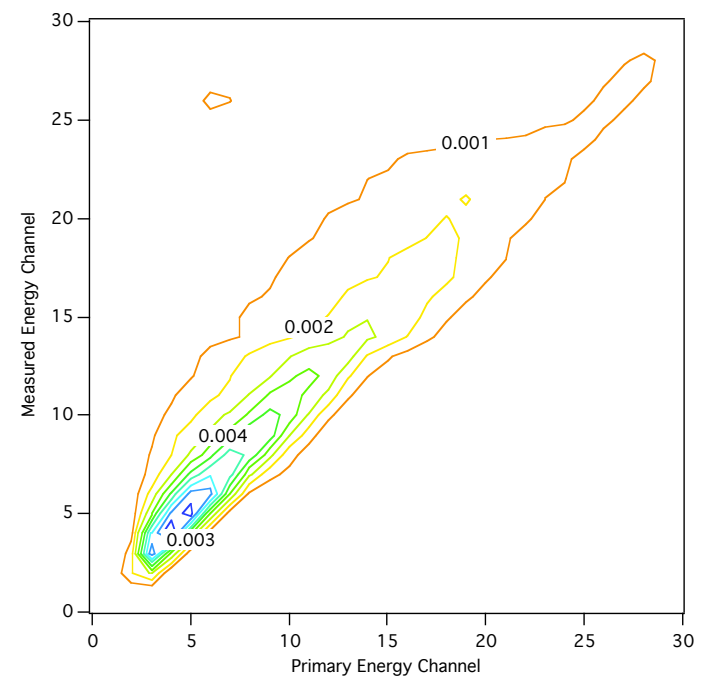

Figure 8. Neutron energy resolution of FNIT from Monte Carlo simulations, determined by using only double n-p scatter events. The actual energy of each incident neutron (horizontal coordinate) is plotted against its reconstructed energy (vertical coordinate). Energy units on both axes are $\mathrm{MeV}$.

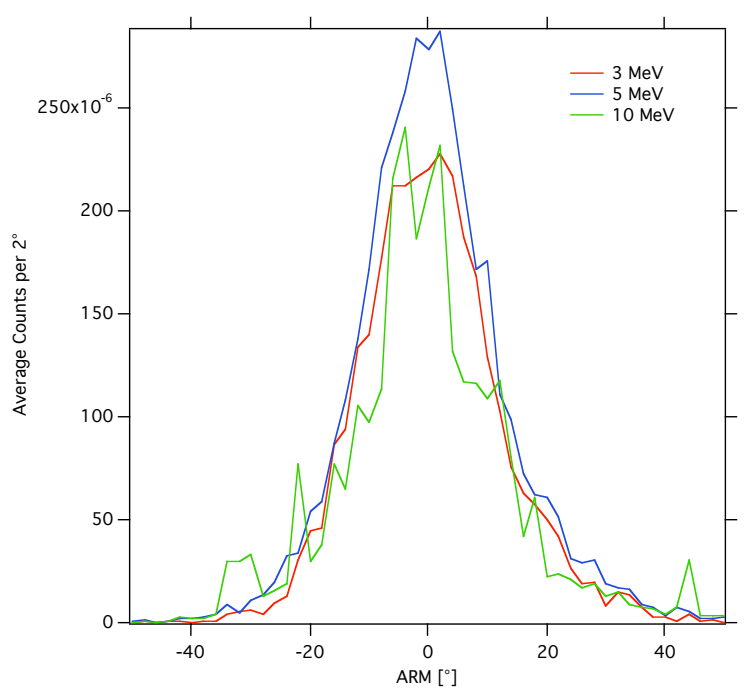

Figure 9. Angular resolution of FNIT for double n-p scatter neutrons at three different energies from Monte Carlo simulations. This histogram represents the distributions of the differences between actual value and reconstructed value of the neutron scatter angle $\theta_{\mathrm{n}}$. Units on the horizontal axis are degrees. 

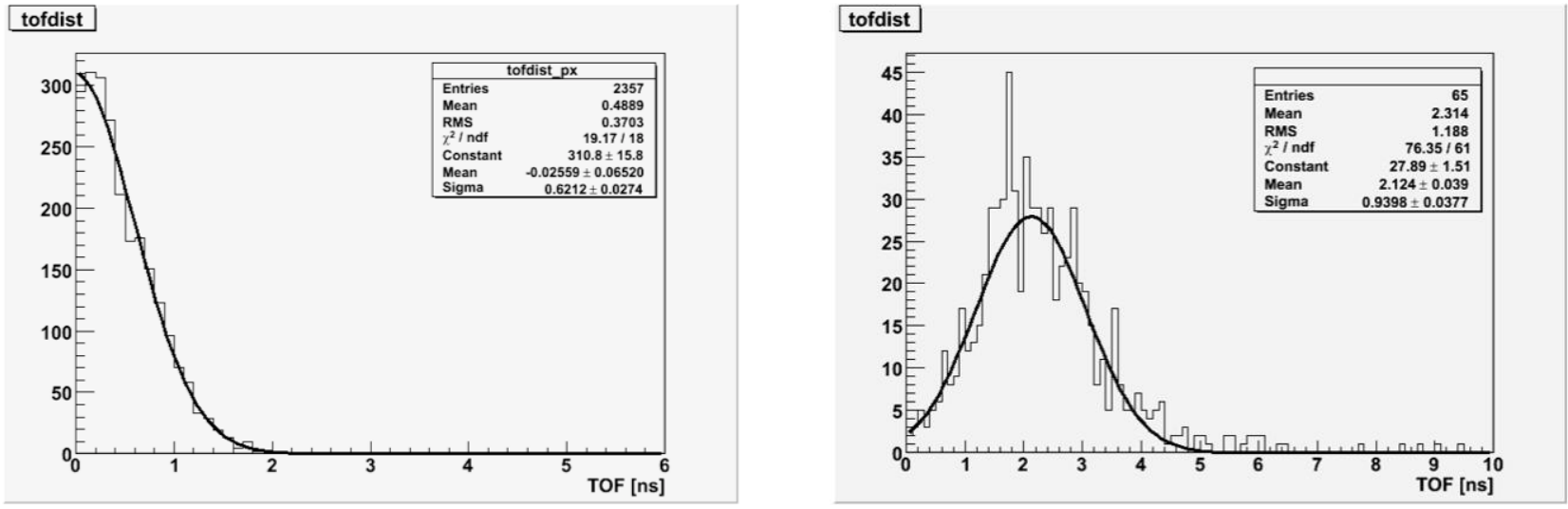

Figure 10. Time of flight resolution in FNIT from Monte Carlo simulations. The ToF distribution of $\gamma$-rays (left histogram) is compared to the ToF of double n-p scatter neutrons with initial energy $\mathrm{E}=10 \mathrm{MeV}$ (right histogram).

One additional obstacle to SNM imaging that needs to be mentioned at this point is represented by background particles. We briefly discussed the background from atmospheric neutrons in Section 2. Imaging will be used to cope with these events, but a full SNM sample plus atmospheric background simulation (i.e. the modeling of a real application scenario) is needed prior to drawing conclusions on FNIT capabilities. However, an additional limitation of FNIT, intrinsic in using plastic scintillator and relying on the n-p elastic scattering interaction, is represented by the response of this detector material to all types of charged particles, as well as to $\gamma$-rays that produce Compton-scatter electrons, mimicking a neutron generating recoil protons. At sea level, the atmospheric cosmic-ray flux, consisting primarily of muons, equals on average $2.4 \times 10^{-2}$ particles $/ \mathrm{cm}^{2} \mathrm{~s}^{14}$. This figure is only a few times larger than the atmospheric neutron flux from Section 2, but the efficiency of plastic scintillator for muons is nearly $100 \%$. Therefore, in the FNIT instrument the trigger rate from atmospheric muons can be expected to be over an order of magnitude larger than the trigger rate from atmospheric neutrons. The need for an active veto shield around FNIT, to reject all energetic charged particles, will be assessed in the future and additional simulations may be needed to determine the effect on such a shield on neutron sensitivity.

Finally, $\gamma$-rays cannot be vetoed with an active shield, but the ToF of scattered neutrons between detector modules may be used for this purpose. Namely, the velocity of $\gamma$-rays is the speed of light $c$, while e.g. 5 $\mathrm{MeV}$ neutrons have a velocity of $\sim 0.1 \mathrm{c}$. Considering the time resolution measured in Section $4(\delta \mathrm{t}=0.6 \mathrm{~ns})$, the vertical pitch of the FNIT tower was fixed at $4.5 \mathrm{~cm}$ (i.e. a $3.0 \mathrm{~cm}$ spacing between detector modules was chosen), in part to reject $\gamma$-ray events and in part to accurately determine the velocity of the scattered neutron, used in computing the energy of incident neutrons. Figure 10 compares the simulated ToF distributions of $\gamma$-rays and 10 $\mathrm{MeV}$ incident neutrons. Distribution broadening from the $\delta t=0.6 \mathrm{~ns}$ resolution was incorporated in these simulations. The $\gamma$-ray / neutron separation at this energy is fair and it improves as the energy of the neutron becomes lower. Still, after evaluating the actual $\gamma$-ray background through an application scenario simulation, it may become necessary e.g. to

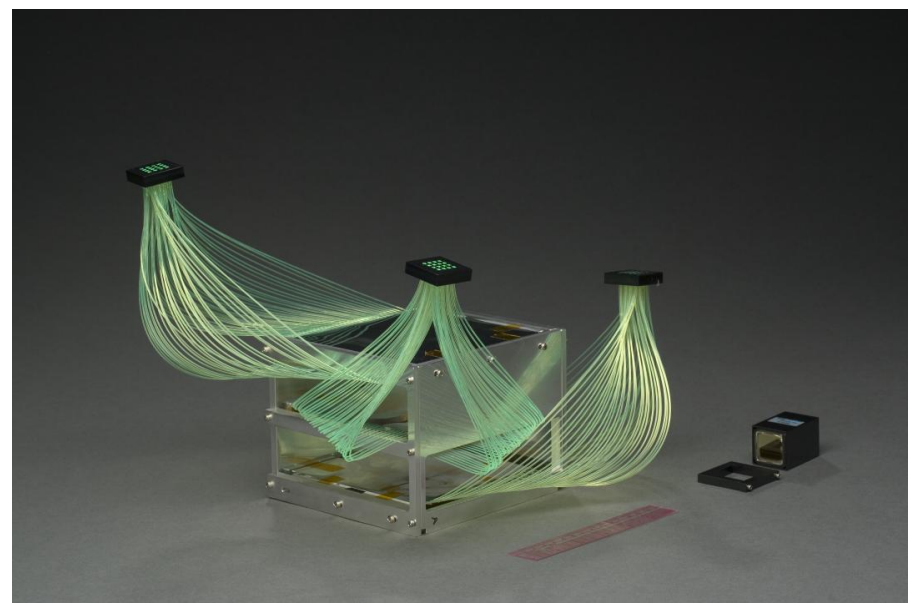

Figure 11. FNIT SM1 setup. Three detector modules are assembled on top of each other in this tower structure. 

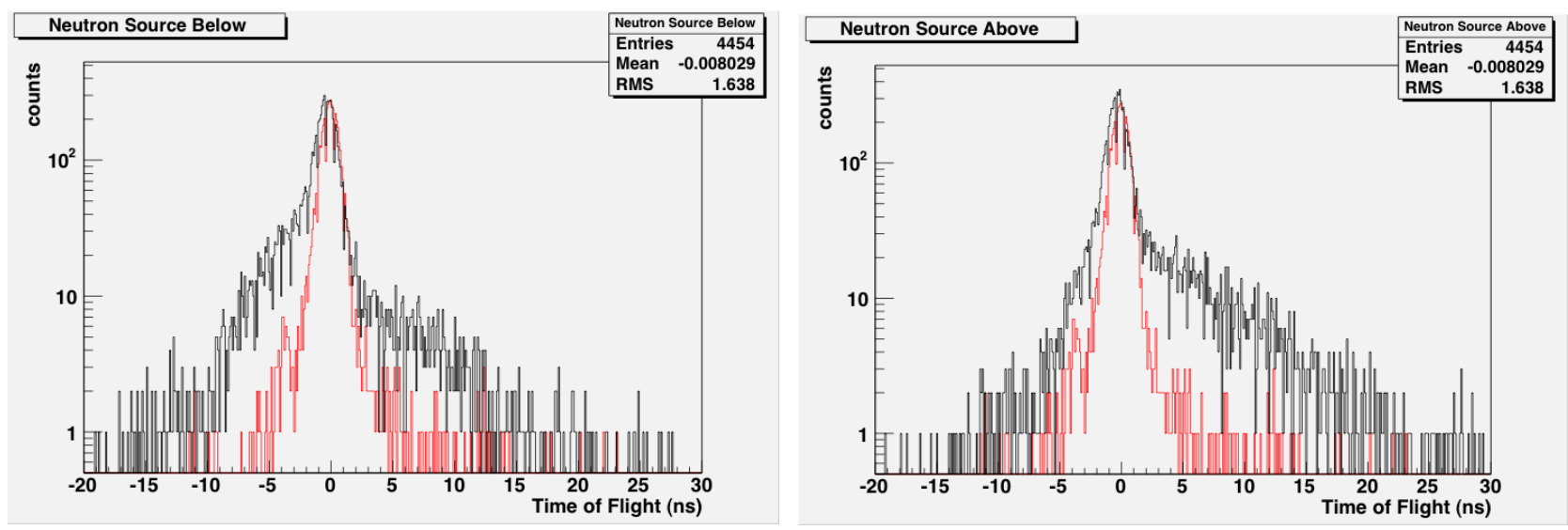

Figure 12. Time of flight resolution in FNIT SM1 from lab data. Three sets of data were acquired: background events only (shown in red on both histograms), background + Am-Be neutron source beneath FNIT SM1 (black distribution in left histogram) and background + Am-Be neutron source on top of FNIT SM1 (black distribution in right histogram). Am-Be neutrons are significantly slower than background particles. Therefore, the "tails" in the two black ToF distributions on opposite sides of the background peaks are clearly attributable to neutrons.

increase the vertical pitch of FNIT to achieve a sufficient $\gamma$-ray rejection power. We should note that $\gamma$-ray detection capability may also present an advantage, since unshielded SNM samples produce copious $\gamma$-ray emissions in addition to fission neutrons.

\section{SCIENCE MODEL ONE}

The Science Model One (SM1) of FNIT was recently assembled and testing with lab sources is currently underway. One goal for SM1 is to correctly reproduce the image and energy spectrum of a neutron point source, most likely one of the radiation sources (e.g. Am-Be) commonly available in the lab.

The structure of SM1 follows the FNIT design layout shown in Figure 4. The detector modules were manufactured according to the specifications from Section 4 and the $4.5 \mathrm{~cm}$ vertical pitch was used. However, SM1 consists of only three detector modules rather than eight. While the overall efficiency is reduced as a result, especially for triple $n-p$ scatters, the sensitivity of SM1 is not affected by this descoping and three detector modules are fully sufficient to characterize the performance of the full FNIT instrument in a lab environment. Figure 11 shows a photograph of the FNIT SM1 detectors taken prior to them being sealed in a light-tight box to undergo testing.

Signals from the three detector modules are seen by three MAPMTs. NIM electronics is used to process these signals, which result in a total of 54 data channels per event, namely:

- $16 \times 3=48$ anode channels from the three MAPMTs;

- one dynode signal from each MAPMT to feed the ToF circuitry; and

- one additional dynode signal per MAPMT to measure the total light yield from each module and for trigger.

These pulses are digitized by two CAEN 785 ADCs and one $775 \mathrm{~N} \mathrm{TDC}^{15}$ installed in a VME crate. A WIENER VMUSB module ${ }^{16}$ functions as the crate controller, connected through a USB port to the desktop computer used for data acquisition (DAQ). The coincidence of either two or three dynode signals is used to trigger the SM1 apparatus. We tested this DAQ setup with a pulse generator and were able to handle a $10 \mathrm{kHz}$ trigger rate, which by far exceeds our lab needs and also the data rate envisioned for any potential application scenario.

At present, the SM1 is positioned vertically (as in Figure 4) and cosmic-ray muons are being used for calibrations and matching of the pulse intensities in all data channels. As a preliminary demonstration of the potential of FNIT SM1, this prototype instrument was irradiated with an Am-Be neutron source. This source produces a neutron energy spectrum comparable to the fission spectrum from SNM that was shown in Figure 1 and has a peak value of a few 
$\mathrm{MeV}^{17}$. In addition, ${ }^{241} \mathrm{Am}$ emits $60 \mathrm{keV} \gamma$-rays. The Am-Be source was placed alternatively on top and underneath SM1, the hardware thresholds were set to cut the low-energy $\gamma$-rays and SM1 was operated in double-coincidence trigger mode. The signature of neutrons from the Am-Be source is clearly visible in the ToF spectra from these two setups. The observed distributions, which should be regarded only as an initial, very preliminary result, are shown in Figure 12 .

\section{CONCLUSIONS}

The goal of the FNIT project is to demonstrate the feasibility of an imaging neutron detector for SNM identification. At present, the design specifications for the basic detector module used in the FNIT tower structure are frozen for the SM1 studies and the Monte Carlo simulation code is fully operational. The SM1 of FNIT has been assembled and is now undergoing performance testing. A full set of lab tests on SM1 needs to be completed to characterize the efficiency and sensitivity of this instrument. Additional Monte Carlo simulations are needed to model the signal from SNM samples blended with background event fluxes representative of a realistic application scenario. Algorithms for neutron imaging need to be expanded and adapted for SNM identification. Finally, the imaging of an actual neutron lab source with the FNIT SM1 has to be demonstrated. In conclusion, a large amount of work still needs to be done prior to considering the upgrade of FNIT into a field-deployable device. Nevertheless, the results achieved so far clearly show that FNIT is a very promising state of the art instrument with a unique potential to develop into an efficient SNM detector.

\section{ACKNOWLEDGMENTS}

We would like to acknowledge the financial support for the FNIT project by our funding agencies: The DoE (contract DE-FG52-04NA25687) and NASA (grant NAG5-13519) in the United States and the Swiss National Science Foundation (grant 200020-105435/1) in Switzerland.

\section{REFERENCES}

1. G. F. Knoll, Radiation Detection and Measurement, 3rd ed., New York: Wiley, 2000.

2. [Online] http://lws.gsfc.nasa.gov/missions/sentinels/sentinels.htm

3. N. Nereson, "Fission Neutron Spectrum of Pu ${ }^{239}$," Phys. Rev., vol. 88, pp. 823-824, 1952.

4. M. R. Moser, J. M. Ryan, L. Desorgher, and E. O. Flückiger, "Atmospheric Neutron Measurements in the 10-170 MeV Range," Proc. $29^{\text {th }}$ Int. Cosmic Ray Conf., SH35, pp. 101-104, 2005.

5. D. Slaughter, et al., "Detection of special nuclear material in cargo containers using neutron interrogation", Lawrence Livermore National Lab, UCRL-ID-155315, 2003.

6. [Online] http://cossc.gsfc.nasa.gov/

7. H. Debrunner, J.A. Lockwood, J.M. Ryan, M.L. McConnell, V. Schönfelder, H. Aarts, K. Bennett, and C. Winkler, "Neutrons fron the 15 June 1991 Solar Flare", Proc. $23^{\text {rd }}$ Int. Cosmic Ray Conf., vol. 3, pp. 115-118, 1993.

8. U. Bravar, P.J. Bruillard, E.O. Flückiger, A.L. MacKinnon, J.R. Macri, P.C. Mallik, M.L. McConnell, M.R. Moser, and J.M. Ryan, "Imaging solar neutrons below $10 \mathrm{MeV}$ in the inner heliosphere", Proc. SPIE, vol. 5901, pp.141$150,2005$.

9. U. Bravar, P.J. Bruillard, E.O. Flückiger, J.R. Macri, M.L. McConnell, M.R. Moser, J.M. Ryan and R.S. Woolf, "A Position Sensitive Plastic Scintillator Detector for Fast Neutron Imaging", submitted to IEEE Trans. Nucl. Sci., 2006.

10. [Online] http://www.photonic.saint-gobain.com/

11. [Online] http://www.hamamatsu.com/

12. S. Agostinelli, et al., "GEANT4: A Simulation Toolkit", Nucl. Inst. Meth., vol. A506, pp. 250-303, 2003.

13. R.S. Miller, J.R. Macri, M.L. McConnell, J.M. Ryan, E. Flückiger, and L. Desorgher, "SONTRAC: An imaging spectrometer for MeV neutrons", Nucl. Inst. Meth., vol. A505, pp. 36-40, 2003.

14. P.K.F. Grieder, Cosmic Rays at Earth, Amsterdam: Elsevier, 2001.

15. [Online] http://www.caen.it/

16. [Online] http://www.wiener-us.com/

17. Compendium of Neutron Spectra and Detector Responses for Radiation Protection Purposes, Vienna: IAEA, 2001. 